Castillo s. y cols.

Rev. Chil. Pedlatr. 65 (4); 210-214, 1994

\title{
Alteraciones cromosómicas en niños referidos para estudio citogenético
}

\author{
Silvia Castillo T.'; Lorena Tobella P.2; \\ Samuel Salazar C. ${ }^{2}$; Vera Daher N.2; Patricia Sanz C. ${ }^{\text {; }}$ \\ Fermina López G. ${ }^{3}$; Cecilia Villaseca $\mathrm{G}^{3}$ \\ Chromosomal abnormalities in infants submited for cytogenetic study

\begin{abstract}
From year 1981 throughout 1993 we hove perlormed 1473 caryolipes in palients aged 0 to 15 years. Reference diagnosis were Down's syndrome $\{30,8 \%)$, mullipte malformations $(19,1 \%)$, psychomotor relardation $(10,5 \%)$. Turner's syndrome $\{8 \%$, abnormol sexual development $[7.6 \%$, short stalure $[7,1 \%$, speech development disturbances $[2 \%)$ and miscelaneous syndromes $(14,9 \%)$. The elficiency of the investigation for chromesonal abnormalities varied accordingly io elinical diagnosis: Down's syndrome $(94.0 \%)$, mulliple molformalions $(24.8 \%$, psychomotor relardation $(13.4 \%$, Turner s syndrome (55.0\%), abnormal sexual developmenl [13.4\%), speech distubances (10.0\%) and other syndromes [9.1\% promotes the search of non chromosomal efiologies in opparently similar phenotypes, and it represents an important contribution lo genelic counsel for affected tomilies.
\end{abstract}

(Key words: Chromosomes, human, abroumalilies, cylogenelics, kariotyping!

Las alteraciones cromosómicas son responsables de proporciones significativas de los casos de pérdidas de productos de la concepción, anomalías congénitas y retardo mental, así como también juegan un importante rol en la patogenia de las enfermedades malignas. Se conocen aberraciones cromosómicas específicas responsables de más de sesenta síndromes. Ellas se detectan en $50 \%$ de los abortos espontáneos del primer trimestre, $6 \%$ de los mortinatos y $0,6 \%$ de los recién nacidos vivos, lo que demuestra que existe una fuerte selección natural eл su contra ${ }^{1}$.

Las anomalías cromosómicas pueden ser numéricas o estructurales y comprometer uno o más autosomas, cromosomas sexuales o ambos simultáneamente. El tipo mús común de alıeración citogenética clínicamente significativa es la aneuploidía o número anormal, por exceso o déficit, de cromosomas, que está siempre asociada

1. Servicio de Genćcica, Departamento de Medicina, Ho5pital Clínico Universidad de Chile.

2. Tecnólogo Mético. Labaratorio de Citogenética. Servicio de Genética, Departamento de Medicina. Hospital Clínico Lniversidad de Chile.

3. Becada Genérica Médica, Ministerio de Salud. a trastornos físicos o mentales. Las anomalías estructurales resultan de fracturas y reconstitu. ciones cromosómicas aberrantes, que pucden ser balanceadas o desbalanceadas (si se ha perdido 0 ganado información genética), causando -en este caso- nn desarrollo anormal del individuo. Hay deleciones (borramientos o pérdidas de una parte o el total de un cromosoma), duplicaciones, cromosomas en anillo (figura 1), jsocromosomas, isodicéntricos, inversiones y translocaciones robertsonianas y recíprocas ${ }^{2,3}$.

Los fundamentos clăsicos para solicitar análisis cromosómico son la comprobación de anomalia citogenética cuando se sospecha por las manifestaciones clínicas (por ejemplo, sindrome de Down), la búsqueda de la causa de malformaciones congénitas múltiples, alteraciones en la diferenciación sexual, talla baja, retardo psicomotor o mental y trastomos en el desarrollo del lenguaje en niños afectados ${ }^{4}$.

El propósito de esta comunicación es describir los resultados y el rendimiento de los estudios cromosómicos realizados a pacientes pediátricos referidos al laboratorio de citogenética de la Universidad de Chile en un período de doce años. No se incluyen los exámenes rcalizados por neoplasias hematologicas, por tratarse de afec- 


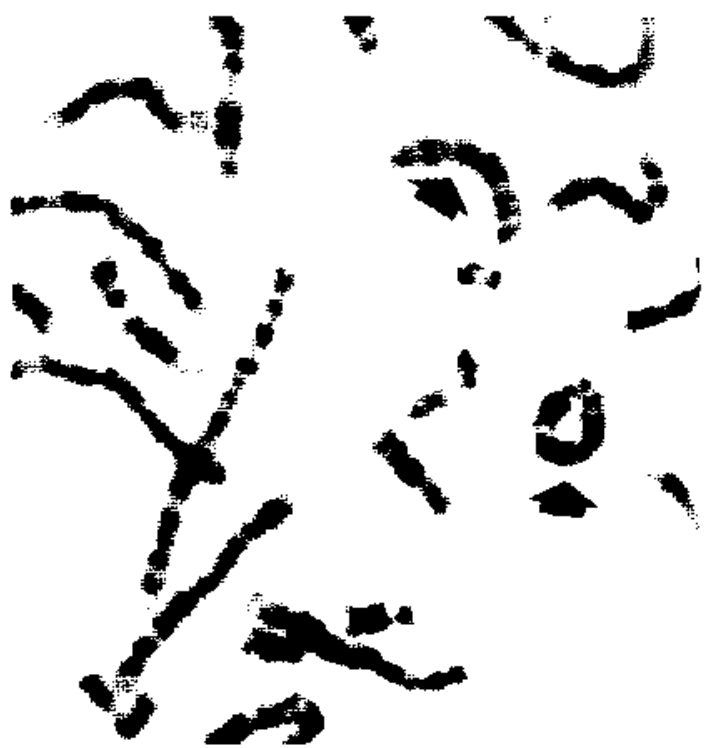

Figura: Microfotografía de parte de los cromosumas de una célula de una pacientes con síndrome de Tumer. Las flechas indican un cromosoma $X$ en anillo y otro cromosoma $\mathrm{X}$ normal.

ciones de origen localizado (en la mćdula ósea) con alteraciones restringidas a ese sistema y de trascendencia diferente.

\section{Pacientes y Método}

Mil cuatrocientos setenta y tres niños, entre recién nacidos y la edad de 15 años, fucron referidos al laboralorio de citogenetica del Hospital Clínico de la Universidad de Chile desde servicios de salud estalales o privados de Santiago y el resto del paŕs, entre enero de 1981 y agosto de 1993. Los diagnósticos de referencia fucron: síndrome de Down en $453(30,8 \%)$, malformaciones múltiples $282(19,1 \%)$, retraso psicomotor $155(10,5 \%)$, sindrome de Tumer $137(9.3 \%)$. alteraciones del desarrollo sexual $112(7,6 \%)$, talla baja 104 $(7,1 \%)$, trastorno del desarrollo del ienguaje 30 (2\%) y síndromes diversos 219 (14,9\%).

A todos los pacientes se les cxtrajo una muestra de 1 a 5 $\mathrm{ml}$ de sangre venosa periférica en una jeringa con heparina. Los cromosomas se obiuvieron mediante culivo de linfo. citos en medio especial con nutrientes y estimulantes de lis mitosis a $37^{\circ} \mathrm{C}$ por 72 horas. En la cosecha se recuperaron las células en proliferación, se sometieton a un choque hipotónico y se fijaron sobre un portaobjeto para proceder a diversas técnicas de tinción y definición de bandas (bandco)" Para componer el cariotipo se examinaron con microscopio 30 céluias con bandeo $G$ (promedio) y se obtuvieron al menos tres microfotografías en cada caso. Para expocsar el diagnóstico cilogenético se empleó la nomenclatura del sistema intemacional ISCN ${ }^{6}$.

\section{Resultados}

El rendimiento del estudio citogenético en los niños analizados fue de 623 exámenes altcrados cn cl total de 1473 (42,3\%). Entre 453 pacientes enviados con diagnóstico de síndrome de Down, los exámenes eran normales en 27. De Ios 426 cariotipos alterados, en 381 había trisomía 21 libre, en 25 se trataba de mosaicos con líneas celulares normales, en 12 de translocaciones robertsonianas: en seis casos $t[21 ; 2 t]$, en cuatro $t[14 ; 21]$, en los restantes $t[13 ; 21]$ y $t[21 ; 22]$ respectivamente. En cuatro pacientes con estc diagnóstico se detectó otro tipo de alteraciones cromosomicas: $46, \mathrm{XX} / 47, \mathrm{XX}+\mathrm{frag} ; 46, \mathrm{XX} /$ altcraciones múltiples; $46, X Y$ 6p + y 46, XY del (18) respoctivamente.

En $70(25 \%)$ de los niños referidos por malformaciones múltiples sc detectaron alteracioncs cilogenéticas. La más frecuente era la trisomía $18(38,6 \%)$, seguida por la trisomía $13(14,3 \%)$ y múltiples reordenamientos estructurales (tabla 1 ).

El retraso psicomotor es un diagnóstico frecuente en la indicación del estudio citogenético; su rendimiento fuc, sin embargo, sólo $13,5 \%$ y entre los hallazgos predominaron las alteraciones estructurales desbalanceadas (tabla 2).

En 137 pacientes se sospechaba sindrome de Turner y en $56,9 \%$ de ellos se confirmó este diagnóstico (tabla 3). Se observaron alteraciones numéricas; principalmente monosomia del cromosoma $\mathrm{X}$ y diversas alteraciones estructurales en combinación con líneas cclulares normales o anormales. Quince pacientes con alteraciones

\section{Tabla 1}

Resultados citogenéticos en niños con malformaciones múltiples

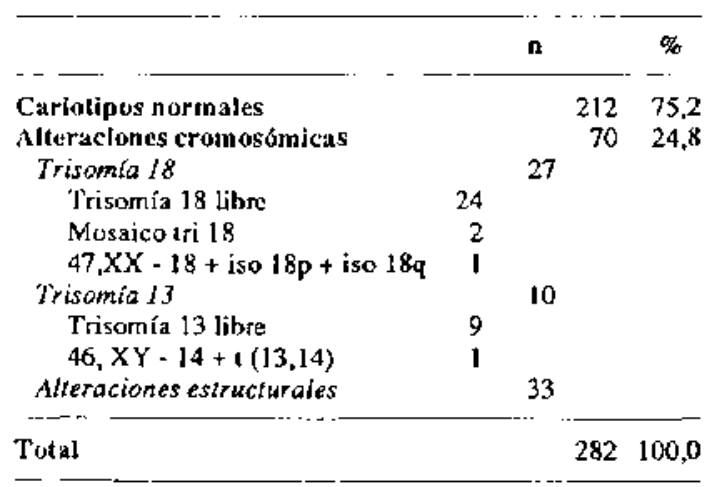


Tabla 2

Resultados citogenćlicos en niños con retraso psiconotor

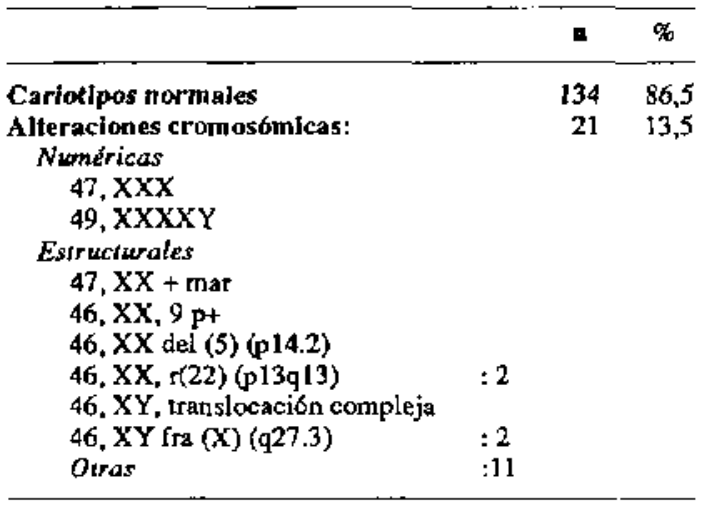

Total

155100,0

Tabla 3

Resultados citogenéticos en casos de síndrome de Tumer

\begin{tabular}{|c|c|c|c|c|}
\hline & & & $\mathbf{n}$ & $\not{k}$ \\
\hline Cariotjpos normales & & & 59 & $43, ?$ \\
\hline Alteraclones cromosómicas: & & & 78 & 56,9 \\
\hline $45, X$ & & 34 & & \\
\hline Mosaicos & & 11 & & \\
\hline $45, X / 46, X X$ & 6 & & & \\
\hline $46, \mathrm{XX} / 47, \mathrm{XXX}$ & 1 & & & \\
\hline $45, X / 46, X X / 47, X X X$ & 1 & & & \\
\hline $45, X / 47, \mathrm{XXX}$ & 3 & & & \\
\hline Atseraciones estructurales & & 27 & & \\
\hline $45, X / 46, X$ iso $X q$ & 7 & & & \\
\hline $45 \times / 46, X X / 46 \times X$ iso $X_{q}$ & 7 & & & \\
\hline $46, X$ iso $X q$ & 3 & & & \\
\hline $45, X / 46, X r(X)$ & 3 & & & \\
\hline Otras & 7 & & & \\
\hline Otras alleractiones & & 6 & & \\
\hline $46, X X$ fra $(8)$ & & & & \\
\hline $46, X \times \mathrm{t}(5 ; 6)$ & & & & \\
\hline 46. XX, del (4) (q32) & & & & \\
\hline $45, X / 46, X Y$ & & & & \\
\hline 46, XX/alteraciones múltiples & 2 & & & \\
\hline
\end{tabular}

Total

$137 \quad 100,0$

cromosómicas fueron referidos al estudio por esLatura baja. En 89 niños ( 8 varones) enviados por talla baja, el cariotipo fue normal excepto en un paciente con 46, XY 6q+. Entre los niños derivados por alteraciones en el desarrollo sexual habia $15(13,4 \%)$ cuyos cariotipos estaban alierados, la mayoría por polisomías (otros con alteraciones estructurales) del cromosoma $X$.

Dentro de un estudio dirigido a niños con trastornos en el desarrollo del lenguaje, se encontró $10 \%$ de alteraciones cromosómicas estructurales. En el heterogéneo grupo de síndromes diversós se incluyen malformaciones aisladas 0 , cn ocasiones, la más severa o relevante presente en el paciente. En la tabla 4 se muestran los 13 diagnósticos que correspondicron a alteraciones cromosómicas en este grupo.

\section{Comentario}

El estudio cromosómico sirve para identificar la causa de ciertos casos de un buen número de afecciones, cntre ellas, malformaciones múltiples, retardo mental, alteraciones en el desarrollo se$x$ uall ${ }^{7}$, etc. Sin embargo, sa rendimiento depende del tipo de trastorno clínico. En esta casuística, el alto porcentaje de alteraciones detectadas se debc en gran medida a que un tercio de la muestra era de niños con síndrome de Down, donde ocurre la mayor concordancia entre los hechos clínicos y citogenéticos.

A pesar de que el diagnóstico clínico de sindrome de Down es relativamente fácil y certero, es importante rcalizar el estudio cromosómico en todos ellos ${ }^{8}$, pues confirma la hipótesis o la descarta en absoluto, como ocurrió en un número no despreciable de pacientes con cariotipo normal u otros defectos. También permite detectar casos potencialmence heredados, translocaciones -en que cs perentorio estudiar a los progenitores para estimar el riesgo de recurrencia- o coexistencia con otras abcrraciones citogenéticas. En los casos en que se detectan mosaicos, con una línea celular normal además de la alterada, es posiblc dar a los padres la esperanza de una respuesta potencialmente mejor al apoyo kinésico y la estimulación. En la forma más frecuente, la trisomia libre, el riesgo de recurrencia empírico es de $1 \%$ y no se rcaliza estuđio cromosómico en forma rutinaria a los padres. Existen numerosas causas de malformaciones congénitas múltiplcs ${ }^{9}$ y en estos casos existe indicación absoluta para realizar cariotipo. En cfecto, el resultado implica un pronóstico muy negativo en los casos de trisomía 18 y 13 , en quicnes la sobrevida no sucle exceder 120 días $^{10}$. Además, en casos producidos por alteraciones estructurales de los cromosomas 
Tabla 4

Resultados citogenéticos en sindromes diversos

\begin{tabular}{|c|c|c|}
\hline $\begin{array}{l}\text { Cariotipos nomales } \\
\text { Cariotipos alterados }\end{array}$ & $\begin{array}{l}152 \\
13\end{array}$ & \\
\hline Diagnóstico & Cariolipo & $\mathbf{n}$ \\
\hline nlec & 46, XY, inv (6) (p22q27) & $1(6)$ \\
\hline oloboma del iris & \multirow{2}{*}{$46, X X / 46, X X+$ frag } & 1 \\
\hline etinoblastor & & \\
\hline ardiopatía congénita & $\begin{array}{l}46, X X, \text { del }(13)(q 14) \\
45, X\end{array}$ & 2 \\
\hline Hidranencefalia & $47, \mathrm{XXX}$ & 1 \\
\hline Atresia esofágica & $47, X Y+21$ & 1 \\
\hline índrome de Pelers & $46, X Y$ del $(13)\{031)$ & 1 \\
\hline Trisomía 22 & $47, X Y+\operatorname{mar}$ & 1 \\
\hline Disoslosis clejdoctar & $46 . X X+$ frag & \\
\hline Anemia de Fanconi & Fragilidad cromosómica & 2 \\
\hline Morfea lineal extensa & $47, X X X$ & 1 \\
\hline
\end{tabular}

Total

13

(): total de exámenes realizados con el mismo diagnóstico.

derivadas de translocaciones balanceadas en uno de los padres, el riesgo de repetición puede ser del orden de 300 aun $50 \%$. Es más común encontrar alteraciones cromosómicas en niños en que el retraso psicomotor se asocia a ciertas malformaciones o dismorfias. Los pacientes portadores de la misma aberración cilogenélica se parecen mós entre sí que a sus propios hermanos ${ }^{11}$. Es importante estudiar a los padres cuando se detecta en ellos alguna anomalía que pudiera derivar de una alteración sin desbalance $y$, por ende, de la que podrían ser portadores sin repercusión fenotípica psíquica ni física. La dccisión reproductiva posterior pertenece a la pareja, toda vez que ha sido objetivamente informada de sus riesgos.

El diagnóstico de síndrome de Turner sólo debicra ser hecho después de confirmar una anomalía del cromosoma $X$, asociada con alteraciones fenolípicas como talla baja, falla ovárica y una variedad de rasgos dismórficos ${ }^{12}$. El Jiagnóstico diferencial en ninos se debe hacer principalmente con el síndrome de Noonan, que se expresa tanto en mujeres como en varones. En lia mitad de los casos se detecta cl cariotipo caractcrístico 45, $X$ por no disyunción meiótica, en que puede haberse perdido otro cromosoma $\mathrm{X}$ o el $\mathrm{Y}$. En un tercio de los pacientes se encuentra una altcración estructural de uno de los cromosomas $\mathrm{X}$; en las restantes hay mosaicos con líneas celu- lares normales, que podrían evolucionar con mejor desarrollo. La infertilidad asociada a cste cuadro es uno de los mayores motivos de pesar para la familia. Con el desarrollo de técnicas de fertilización asistida con donación de óvulos, estas niñas podrían tener embarazos ${ }^{13}$.

Es discutible si se debe realizar cariotipo en pacientes con síndromes bien definidos, ya que la mayoría cs de origen génico y en ellos sería más bien necesario hacer estudios moleculares ${ }^{14}$. En ciertas ocasiones, aquellos se realizan antes de tener certeza del diagnóslico; en otras, con el objeto de apoyarse en ellos para descartar aberraciones cromosómicas con fenotipo similar. Por azar, pudieran encontrarse alteraciones cromosómicas balanceadas en sindromes delinidos, que permitirian hacer hipótesis acerca de Ia localización del gen en ese segmento ${ }^{15}$. Se ha sugerido que la asociación de dos trastornos mendelianos, por su baja frecuencia, sería una indicación para estudio cromosómico, por la posibilidad de detectar acercamientos de segmenlos normalmente alejados. En la casuística que se comenta, algunas alteraciones parecen explicables por un diagnóstico de referencia escueto, como atresia esofágica en un síndrome de Down o cardiopalía congénita en dos pacientes con síndrome de Tumer. Otras parecen asociaciones fortuitas o hallazgos poco habituales. El estudio cilogenético en los nifoos es, en primera instancia, de importancia para el diagnóstico. Permite ocasionalmente establecer, en grandes líneas, pronósti$\cos$ de sobrevida o de desarrollo. Su mayor trascendencia está en la posibilidad de dar consejo genélico, contribuyendo a establecer la causa y el riesgo de recurrencia de una afección en la familia, así como la posibilidad de tener hijos $\operatorname{sanos}^{16}$.

\section{Resumen}

Entre tos affos 1981 y 1993 se realizaron 1473 cariogramas en pacientes de 0 a 15 años en un centro de estudios citogenćlicos de la Universidad de Chile. Los diagnósticos de referencia fueron sindrome de Down (30.8\%), malformaciones múltiples $(19,1 \%)$, retraso psicomotor $(10,5 \%)$, sindrome de Tumer $(9,3 \%)$, alteraciones del desarrollo sexual $(7,6 \%)$, talla baja $(7,1 \%)$, trastornos del desarrollo de lenguaje $(2 \%)$ y sindromes diversos $(14,9 \%)$. El rendimiento del cxamen 
varía según la categoría clínica del diagnóstico: síndrome de Down (94\%), malformaciones múltiples $(24,8 \%)$, retraso psicomotor $(13,4 \%)$, síndrome de Turner $(56,9 \%)$, alteraciones del desarrollo sexual $(13,4 \%)$, trastornos del lenguaje $(10 \%)$ síndromes diversos $(9,1 \%)$. El estudio cromosómico permite comprobar una hipótesis clínica estableciendo el diagnóstico o, si da resultados normales, puede obligar a estudiar otras etiologías, no cromosómicas, que cxpliquen los uastornos del paciente. El objetivo final es contribuir al consejo genético óptimo para la familia afectada.

(Palabras clave: cromosomas, humanos, anomalías, cariotipo.)

\section{Referencias}

1. Gelehrter ThD, Collins FS: Principles of Medical Geneics. Williams and Wilkins, Balcimore, Hong Kong, London, Sydney, 1990.

2. Borgaonkar DS: Chromosomal variation in man: a calalog of chromosomal variants and anomalies. Sth Edition A.R. Liss. Inc. New York 1989.

3. Rooney DE, Czepulkowski BH: lluman Cytogenelics: a practical approach IRL Press, Oxford, Washington DC. 1987.

4. de Grouchy J, Turleau C: Clinical Atlas of Human Chromosomes $2^{\text {td }}$ Edition, Johr Wiley and Sons. Inc. 1984. New York, Chichester, Brisbanc. Toronto. Singapore.
5. Dutrillaw B, Couturier $J$ : La practique de l'analyse chremosomique. Techniques de laboratoire. Masson Paris, New York, 1981.

6. ISCN: An International System for Human Cytogenetic Nomenclature Birth Defects: Original Articie Series vol 2I, N 1,1985 . March od Dimes, Birth Defects Foundation, New York, U.S.A

7. Tortrealba I: Trastomos en la diferenciación sexual. Rev Chil Pedjatr 1993; 64: 23-27.

8. Astete C, Youlton R, Costillo S, Be C, Daher V: Análisis clínico y citogenético en 257 casos de síndrome de Down. Rev Chil Pedialr 1991; 62: 99-102.

9. Nazer J, Castillo S, Cifuentes L, et al.: Frecuencia de malformaciones congénitas en Chile. Rev Med Chil 1989; 117:110-118.

10. Buyse ML: Birth Defects Encyclopedia Center for Birth Defects Information Services. Inc., Dover, M.A., U.S.A. 1990.

11. Jones KL: Smith's Recognizable Patterns of Human Malformations $4^{\text {th }}$ Edition WB Saunders Philadelphia, 1988.

12. Rosenfeld RG: Tumer syndrome: A guide for physicians. The Tumer's Syndrome Saciety. Mason Medical Communications, Inc., Stanford, California 1989.

13. Sauer MV. Paulson RJ, Macaso TM. Francis MM, Lobo $R$ : Oocyte and pre-embryo donation to women with ovarian failure: an exended clinical triai. Fertil Steril 1991: 55: 39.43.

14. MC Kusick VA: Mendelian Inheritance in man. $10^{\text {b }}$ Edition Johns Hopkins Press, Baltimore 1992.

15. Castillo S, Beca JP, Sáez R, Geldres V: Balanced pericentric inversion 8 ( $(23 q 13)$ in a child with rhizomelic chondrodysplasia punctata and his mother. Clin Genet 1991: 40: $247-248$.

16. Castillo S: Conscjo genético. Rev Chil Pediatr 1993; 64: 34-37. 